\section{Diabetic retinopathy progression and visual outcome after phacoemulsification in South-Asian and Afro-Caribbean patients with diabetes}

S Chatterjee, V V Savant and P Stavrou

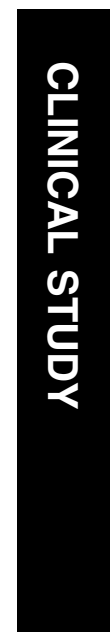

Conclusions Afro-Caribbeans may be at a higher risk of progression of retinopathy or maculopathy after phacoemulsification. Eye (2004) 18, 575-579. doi:10.1038/sj.eye.6700747

Published online 9 January 2004

Keywords: diabetic retinopathy; South-Asian; Afro-Caribbean; phacoemulsification

\section{Introduction}

The prevalence of diabetes is estimated to be $1-3 \%$ in the United Kingdom. ${ }^{1}$ In Western countries, diabetes is the most common risk factor for cataract. ${ }^{2}$ Cataract is the principal cause of legal blindness in older onset diabetes and the second most common cause in younger onset diabetes. ${ }^{3}$

Cataract surgery in diabetic patients is associated with a higher incidence of postoperative complications including fibrinous uveitis, ${ }^{4}$ posterior capsule opacification, ${ }^{5}$ anterior segment neovascularisation, ${ }^{6}$ cystoid macular oedema, ${ }^{7}$ and worsening of diabetic retinopathy and maculopathy. ${ }^{8}$ While some studies have described increased progression of diabetic retinopathy and maculopathy following phacoemulsification cataract surgery, others have not. ${ }^{9-11}$

Recent studies have suggested differences in the prevalence of complications, including retinopathy between various races. ${ }^{12,13}$ Chowdhury and Lasker ${ }^{12}$ in a study of 292 newly diagnosed early-onset type II diabetic patients, including 165 South-Asians, found a higher prevalence of macrovascular and
Birmingham and Midland Eye Centre, UK

Correspondence: P Stavrou Birmingham and Midland Eye Centre, City Hospital NHS Trust, Dudley Road Birmingham B18 7QU, UK Tel: + 441215076854 Fax: +441215076853 E-mail: panagiotastavrou@ hotmail.com

Received: 13 February 2003 Accepted in revised form: 6 August 2003 Published online: 9 January 2004 
microvascular disease including retinopathy and nephropathy in South-Asians. Data from the Cardiovascular Health Study ${ }^{13}$ suggested a higher prevalence of retinopathy in black Americans compared to white Americans with diabetes. However, the UK Prospective Diabetes Study ${ }^{14}$ (82\% white Caucasian, $10 \%$ Asian of Indian origin, and $8 \%$ Afro-Caribbean) found the prevalence of microvascular complications including retinopathy at diagnosis of type II diabetes to be similar between white Caucasian, Asian, and Afro-Caribbean patients. At present, there is no information in the literature about progression of retinopathy or maculopathy in patients from ethnic minorities. The purpose of this study was to assess retinopathy or maculopathy progression and visual outcome after phacoemulsification in South-Asian and Afro-Caribbean patients with diabetes.

\section{Patients and methods}

A retrospective case note analysis was performed on patients with diabetes undergoing cataract extraction by phacoemulsification between April 2000 and December 2001. The inclusion criteria were: (a) South-Asian (Pakistani, Indian, or Bangladeshi origin) or AfroCaribbean ethnicity, (b) monocular cases with a minimum postoperative follow-up of 6 months, and (c) binocular cases with an interval of at least 6 months postoperative follow-up in the first eye. Of the 51 case notes reviewed 30 eyes fulfilled the study criteria. The main cause for exclusion from the study was short postoperative follow-up, which is due to a high rate of nonattendance in our diabetic clinic and a large number of patients with bilateral cataract in whom the interval between the cataract surgery in the two eyes was less than 6 months.

Preoperative data included age, sex, race, type and duration of diabetes, hypoglycaemic treatment, medical history, ocular history, and Snellen visual acuity. Preoperative glycaemic control was assessed by $\mathrm{HbA}_{1 \mathrm{c}}$ measurement. Digital imaging of both fundi was performed in all patients preoperatively and at each follow-up clinic review. Seven standard photographic fields of the modified Airlie House classification were obtained by a certified medical photographer using a $30^{\circ}$ fundus camera. ${ }^{15}$ The grading was performed by one masked observer (PS). Retinopathy was graded as no retinopathy, nonproliferative retinopathy, preproliferative retinopathy, and proliferative retinopathy, as defined by the Early Treatment Diabetic Retinopathy Study (ETDRS). ${ }^{15}$ Maculopathy was graded as no maculopathy and clinically significant macular oedema, according to the ETDRS criteria. ${ }^{16}$ Postoperatively, patients were reviewed on the 1st day,
2 weeks, 3 months, 6 months, and then every 6 months. Patients who developed either new or recurrent macular oedema within 3 months of surgery underwent fluorescein angiography. Panretinal and macular laser photocoagulation were performed according to ETDRS guidelines. $^{16}$

In patients with dense cataract precluding assessment of fundus preoperatively, the status of retinopathy or maculopathy on the first postoperative date was considered to be the preoperative stage. In monocular cases, the fellow eye was used as the control eye, while in binocular cases, the second eye was used as the control eye until the second cataract procedure was performed. The criteria for progression of retinopathy were: (1) appearance of retinopathy were previously absent, (2) progression from nonproliferative to preproliferative retinopathy, (3) progression from preproliferative to proliferative retinopathy, and (4) development or progression of maculopathy to clinical significance. Symmetric progression of retinopathy or maculopathy was defined as simultaneous progression in both the operated and fellow eyes. Asymmetric progression of retinopathy or maculopathy was defined as progression in the operated eye when compared to the fellow eye.

McNemar's test for paired data was used to compare the number of operated and fellow eyes that developed progression of retinopathy or maculopathy

postoperatively. The student's $t$-test was used to compare the mean duration of diabetes and mean $\mathrm{HbA}_{1 \mathrm{c}}$ in SouthAsian with that of Afro-Caribbean patients. Logistic regression analysis was performed to study the relationship between retinopathy or maculopathy progression and the following factors: race, use of insulin, age over 65 years, and the presence of treated proliferative diabetic retinopathy preoperatively.

\section{Results}

In all, 30 patients per 30 eyes were included in the study (Table 1$)$. The mean $( \pm S D)$ age was $68.9( \pm 10)$ years, range 41-91 years. The male-to-female ratio was $1: 1$. There were 19 South-Asians (Pakistani, Indian, and Bangladeshi) and 11 Afro-Caribbeans. All patients had type II diabetes. The mean $( \pm S D)$ preoperative duration of diabetes was $9.4( \pm 7.6)$ years, range $0.8-30$ years. The mean $( \pm S D)$ postoperative follow-up was $12.4( \pm 6.5)$ months, range 6-36 months. In all, 11 patients required insulin, while 19 patients were controlled by diet or oral hypoglycaemic agents. A total of 20 patients had known systemic hypertension. In 21 patients, only one eye was operated on, while in the remaining nine patients both eyes underwent phacoemulsification (however, only one eye was included in the study). All operations were performed using a standard phacoemulsification 
Table 1 Demographic data

\begin{tabular}{lc}
\hline Number of patients/eyes & $30 / 30$ \\
Mean $( \pm$ SD) postoperative follow-up (months) & $12.4( \pm 6.5)$ \\
Male/female & $15 / 15$ \\
Mean $( \pm$ SD) age (years) & $68.9( \pm 10)$ \\
Ethnicity & \\
$\quad$ South-Asian & 19 \\
$\quad$ Afro-Caribbean & 11 \\
Mean ( \pm SD) duration of diabetes (years) & $9.4( \pm 7.6)$ \\
Treatment regimen & \\
$\quad$ Diet/tablet & 19 \\
Insulin & 11 \\
Coexisting hypertension & \\
$\quad$ Yes & 20 \\
$\quad$ No & 10 \\
Preoperative visual acuity & \\
$\quad \geqslant 6 / 12$ & 09 \\
6/18-6/36 & 08 \\
$\quad \leqslant 6 / 60$ & 13 \\
Monocular/binocular & $21 / 9$ \\
Operated eye & \\
$\quad$ Right eye & 14 \\
Left eye & 16 \\
\hline
\end{tabular}

Table 2 Preoperative status of diabetic retinopathy

\begin{tabular}{lcccc}
\hline & None & NPDR & PrePDR & PDR \\
\hline Operated eye & 22 & 5 & 0 & 3 \\
Control eye & 23 & 5 & 1 & 1 \\
\hline
\end{tabular}

NPDR, nonproliferative diabetic retinopathy; PrePDR, proliferative diabetic retinopathy; PDR, proliferative diabetic retinopathy.

procedure by one surgeon (PS). Of the 30 operated eyes (93.3\%), 28 had cataract extraction by

phacoemulsification, while in the remaining two patients the phacoemulsification was converted to extracapsular cataract extractions due to extension of the anterior capsular rhexis. No other intraoperative complications were noted.

Preoperatively, 22 of the 30 operated eyes $(73.3 \%)$ had no retinopathy, five eyes $(16.3 \%)$ had nonproliferative retinopathy, and three eyes $(10 \%)$ had treated proliferative retinopathy (Table 2 ). In the fellow eyes, 23 of the 30 eyes $(76.6 \%)$ had no retinopathy, five eyes $(16.3 \%)$ had nonproliferative retinopathy, one eye $(3.3 \%)$ had preproliferative retinopathy, and one eye (3.3\%) had treated proliferative retinopathy. Furthermore, 27 of the 30 operated eyes $(90 \%)$ had no maculopathy and three eyes $(10 \%)$ had been treated for clinically significant macular oedema (Table 3). Among the fellow eyes, 26 of the 30 eyes $(86 \%)$ had no maculopathy, and three eyes $(10 \%)$ had been treated for clinically significant macular oedema.

Postoperatively, seven patients $(23.33 \%)$ developed progression of retinopathy or maculopathy in the
Table 3 Preoperative status of diabetic maculopathy

\begin{tabular}{lcc}
\hline & None & CSMO \\
\hline Operated eyes & 27 & 3 \\
Control eyes & 27 & 3 \\
\hline CSMO, clinically significant macular oedema. &
\end{tabular}

operated eye. Five of the seven patients who developed progression or retinopathy or maculopathy were AfroCaribbeans, while two were South-Asians. Therefore, five of the 11 Afro-Caribbeans $(45.4 \%)$ and two of the 19 South-Asians (10.5\%) developed progression of retinopathy or maculopathy during the study period. All seven patients who developed progression of retinopathy or maculopathy suffered from coexisting hypertension.

Of the seven patients who developed progression, five patients $(16.67 \%)$ showed symmetric progression in both the operated and fellow eyes and two patients $(6.67 \%)$ showed asymmetric progression in the operated eye compared to the fellow eye. Four of the five patients who showed symmetric progression were Afro-Caribbeans and one was South-Asian. Of those with asymmetric progression, one was Afro-Caribbean and the other was South-Asian. Three of the five patients who developed symmetric progression progressed from no retinopathy to nonproliferative diabetic retinopathy, one patient suffered progression from nonproliferative to proliferative retinopathy and required panretinal photocoagulation, while the fifth patient required macular focal photocoagulation for progression of maculopathy. In the two patients who developed asymmetric progression in the operated eye, one developed chronic postoperative uveitis and rubeosis iridis, while the other one progressed from no retinopathy to nonproliferative retinopathy. The patient who developed chronic postoperative uveitis and rubeosis iridis was a 76-year-old Afro-Caribbean female who had been suffering from diabetes for 10 years. Glycosylated haemoglobin $\left(\mathrm{HbA}_{1 \mathrm{c}}\right)$ prior to phacoemulsification was $8.4 \%$. The patient died from cerebrovascular accident 26 months after cataract surgery. Table 4 shows progression of diabetic retinopathy by ethnicity and preoperative diabetic retinopathy status. Pseudophakic macular oedema, confirmed by fluorescein angiography, developed in an Afro-Caribbean patient with nonproliferative diabetic retinopathy preoperatively. This responded well to orbital floor injection and topical steroids, and the visual acuity at 11 months postoperatively was 6/9. No progression of retinopathy was noticed in this patient. 
Table 4 Progression of retinopathy following surgery

\begin{tabular}{lccc}
\hline & $\begin{array}{c}\text { No } \\
\text { progression }\end{array}$ & $\begin{array}{c}\text { Symmetric } \\
\text { progression }\end{array}$ & $\begin{array}{c}\text { Asymmetric } \\
\text { progression }\end{array}$ \\
\hline Ethnicity & & & \\
$\quad$ Asian & 17 & 1 & 1 \\
$\quad$ Afro-Caribbean & 6 & 4 & 1 \\
Preoperative DR & & & \\
status & & & \\
$\quad$ No DR & 19 & 2 & 1 \\
NPDR & 2 & 2 & 1 \\
PrePDR & 0 & 0 & 0 \\
$\quad$ PDR & 2 & 1 & 0 \\
\hline
\end{tabular}

DR, diabetic retinopathy; NPDR, nonproliferative diabetic retinopathy; PrePDR, preproliferative diabetic retinopathy; PDR, proliferative diabetic retinopathy.

Table 5 Logistic regression analysis for retinopathy progression

\begin{tabular}{lcc}
\hline Variable & Odds ratio $(95 \%$ Confidence Interval) & $\mathrm{P}$ \\
\hline Race & $4.32(1,18.63)$ & $0.03^{*}$ \\
Insulin treatment & $3.26(0.75,14.25)$ & 0.09 \\
Age > 65 years & $2.57(0.35,18.39)$ & 0.86 \\
Treated PDR & $1.5(0.26,8.62)$ & 0.85 \\
\hline
\end{tabular}

PDR, proliferative diabetic retinopathy.

*Denotes statistical significance.

Postoperatively, 26 of the 30 operated eyes $(86.67 \%$ ) achieved a final visual acuity $6 / 12$ or better. The visual acuity improved by at least two lines in the Snellen chart in 19 of the 30 operated eyes $(63.3 \%)$. Causes of postoperative visual acuity worse than $6 / 12$ were chronic uveitis with rubeosis iridis (one patient), marked asteroid hyalosis (one patient), and clinically significant macular oedema (two patients).

Overall, seven of the 30 operated eyes $(23.3 \%)$ and five of the 30 fellow eyes (16.6\%) showed progression of retinopathy or maculopathy. There was no significant difference in the number of operated and fellow eyes who developed progression of retinopathy or maculopathy ( $P=0.50$, McNemar's test). The mean $( \pm S D)$ preoperative duration of diabetes in South-Asian patients was $10.4( \pm 8.5)$ years, range $0.8-30$ years, as compared to $7.6( \pm 5.4)$ years, range $1-16$ years, in AfroCaribbean patients. The mean $( \pm \mathrm{SD}) \mathrm{HbA}_{1 \mathrm{c}}$ in SouthAsian patients was $7.8 \%( \pm 1.1 \%)$, range $6.9-9.9 \%$, as compared to $7.5 \%( \pm 1.2 \%)$, range $6.1-9.9 \%$, in AfroCaribbean patients. No statistically significant difference was found in the mean preoperative duration of diabetes and mean $\mathrm{HbA}_{1 \mathrm{c}}$ between South-Asian and AfroCaribbean patients ( $P=0.34$ and 0.28 , respectively, Student's $t$-test).
Logistic regression analysis of the relationship between retinopathy or maculopathy progression and various factors such as race, use of insulin, age over 65 years, and the presence of treated proliferative diabetic retinopathy preoperatively showed that the Afro-Caribbean race was significantly associated with retinopathy or maculopathy progression $(P=0.02)$ (Table 5$)$.

\section{Discussion}

The reported rate of progression of diabetic retinopathy following phacoemulsification in the general diabetic population ranges between 20 and 34\%.9,10,17,18 Mittra et $\mathrm{al}^{10}$ in a retrospective review of 150 eyes of 119 diabetic patients undergoing phacoemulsification reported retinopathy progression in 25\% eyes with 6-10 months postoperative follow-up. In our study, where similar criteria as Mittra et al were used for defining progression of retinopathy, $23.4 \%$ of patients showed either symmetric or asymmetric retinopathy progression at a mean postoperative follow-up of 12.4 months. It is noteworthy that systemic hypertension, a known risk factor for progression of diabetic retinopathy, ${ }_{1}^{19}$ was present in all seven patients in our study who developed symmetric or asymmetric progression.

In our study, asymmetric progression developed only in two of seven patients who developed progression or retinopathy or maculopathy postoperatively. One of the two patients who developed asymmetric progression had a complicated postoperative course with chronic uveitis and development of rubeosis iridis. The other one progressed from no retinopathy to nonproliferative diabetic retinopathy. Our results are similar to those reported by Squirrell et $a l^{9}$ and indicate that phacoemulsification is not associated with acceleration of diabetic retinopathy or maculopathy postoperatively. These findings suggest that symmetric progression following uncomplicated phacoemulsification represents the natural history of the disease, while a complicated postoperative course may be associated with asymmetric progression of retinopathy.

In our study, 26 of the 30 eyes $(86.7 \%)$ achieved a final postoperative visual acuity of 6/12 or better and in 19 eyes $(63.3 \%)$, visual acuity improved by at least two lines in the Snellen chart. Our results compare well with the studies on phacoemulsification in the general diabetic population. Squirrell et $a l^{9}$ reported a postoperative Snellen visual acuity of $6 / 12$ or better in $63 \%$ and improvement of two lines or more in $74 \%$ of 50 patients. Mittra et $a l^{10}$ described a postoperative Snellen visual acuity of at least $6 / 12$ in $62 \%$ and an improvement of two lines or more in $78 \%$ of operated eyes.

In our series, progression of diabetic retinopathy or maculopathy occurred more often in Afro-Caribbeans 
compared to South-Asians $(P=0.02)$. Although we found no statistically significant difference regarding duration of diabetes and $\mathrm{HbA}_{1 \mathrm{c}}$ between the two groups, it is possible that other parameters, which were not examined in this study, such as lipid profiles and socioeconomic characteristics may be related to the outcome. Despite the fact that our study is limited by the small number of patients in each group and its retrospective nature, these results are important when organising ophthalmic care in hospitals serving a large population of ethic minorities like ours.

In conclusion, our results suggest that uncomplicated phacoemulsification in South-Asian and Afro-Caribbean patients with diabetes is associated with a favourable postoperative visual outcome that compares well with the results of phacoemulsification in the general diabetic population. Our findings also suggest that the rate of progression of retinopathy and maculopathy in Asian and Afro-Caribbean patients with diabetes are similar to that reported in the general diabetic population. Largerscale studies are necessary to assess further the evidence of a higher rate of progression in Afro-Caribbeans found in our series.

\section{References}

1 Pozza G, Garancini P, Gallus G. Prevalence and Incidence of NIDDM (Chapter 3). In: Williams R, Papo L, Fuller J (eds). Diabetes in Europe. John Libbey Ltd: London, UK, 1994.

2 Ederer F, Hiller R, Taylor HR. Senile lens changes and diabetes in two population studies. Am J Ophthalmol 1981; 91: 381-395.

3 Klein R, Klein BE, Moss SE. Visual impairment in diabetes. Ophthalmology 1984; 91: 1-9.

4 Hykin PG, Gregson RM, Stevens JD, Hamilton PA. Extracapsular cataract extraction in proliferative diabetic retinopathy. Ophthalmology 1993; 100: 394-399.

5 Ionides A, Dowler JG, Hykin PG, Rosen PH, Hamilton AM. Posterior capsule opacification following diabetic extracapsular extraction. Eye 1994; 8: 535-537.

6 Ulbig MR, Hykin PG, Foss AJ, Schwartz SD, Hamilton PA. Anterior hyaloidal fibrovascular proliferation after extracapsular cataract extraction in diabetic eyes. Am J Ophthalmol 1993; 115: 321-326.
7 Pollack A, Leiba H, Bukelman A, Oliver M. Cystoid macular oedema following cataract extraction in patients with diabetes. Br J Ophthalmol 1992; 76: 221-224.

8 Jaffe GJ, Burton TC, Kuhn E, Prescott A, Hartz A. Progression of nonproliferative diabetic retinopathy and visual outcome after extracapsular cataract extraction and intraocular lens implantation. Am J Ophthamol 1992; 114: 448-456.

9 Squirrell D, Bhola R, Bush J, Winder S, Talbot JF. A prospective, case controlled study of the natural history of diabetic retinopathy and maculopathy after uncomplicated phacoemulsification cataract surgery in patients with type 2 diabetes. Br J Ophthalmol 2002; 86: 565-571.

10 Mittra RA, Borrillo JL, Dev S, Mieler WF, Koenig SB. Retinopathy progression and visual outcomes after phacoemulsification in patients with diabetes mellitus. Arch Ophthalmol 2000; 118: 912-917.

11 Wagner T, Knaflic D, Rauber M, Mester U. Influence of cataract surgery on the diabetic eye: a prospective study. Ger J Ophthalmol 1996; 5: 79-83.

12 Chowdhury TA, Lasker SS. Complications and cardiovascular risk factors in South Asians and Europeans with early-onset type 2 diabetes. QJM 2002; 95: 241-246.

13 Klein R, Marino EK, Kuller LH, Polak JF, Tracy RP, Gottdiener JS et al. The relation of atherosclerotic cardiovascular disease to retinopathy in people with diabetes in the Cardiovascular Health Study. Br J Ophthalmol 2002; 86: 84-90.

14 UK Prospective Diabetes Study. XII: differences between Asian, Afro-Caribbean and white Caucasian type 2 diabetic patients at diagnosis of diabetes. UKPDS. Diabet Med 1994; 11: 670-677.

15 ETDRS report no. 10. Grading diabetic retinopathy from stereoscopic color fundus photographs - an extension of the modified Airlie house classification. Ophthalmology 1999; 98: 786-806.

16 ETDRS study group. Photocoagulation for diabetic macular oedema. Arch Ophthalmol 1985; 103: 1796-1806.

17 Zaczek A, Olivestedt G, Zetterstrom C. Visual outcome after phacoemulsification and IOL implantation in diabetic patients. Br J Ophthalmol 1999; 83: 1036-1041.

18 Antcliff RJ, Poulson A, Flanagan DW. Phacoemulsification in diabetics. Eye 1996; 10: 737-741.

19 UK Prospective Diabetes Study. Tight blood pressure control and risk of macrovascular and microvascular complications in type 2 diabetes: UKPDS 38. Brit Med J 1998; 317: 703-713. 